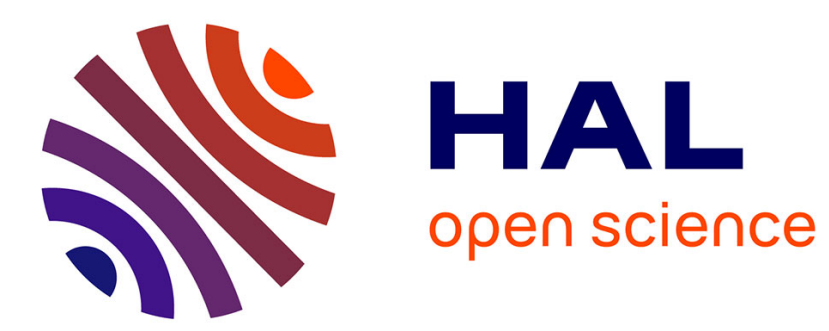

\title{
Diabolo Nanoantenna for Enhancing and Confining the Magnetic Optical Field
}

T. Grosjean, Mathieu Mivelle Mivelle, F.I. Baida, G.W. Burr, U.C. Fischer

\section{To cite this version:}

T. Grosjean, Mathieu Mivelle Mivelle, F.I. Baida, G.W. Burr, U.C. Fischer. Diabolo Nanoantenna for Enhancing and Confining the Magnetic Optical Field. Nano Letters, 2011, 11 (3), pp.1009-1013. $10.1021 / \mathrm{nl} 103817 \mathrm{f}$. hal-00584209

\section{HAL Id: hal-00584209 \\ https://hal.science/hal-00584209}

Submitted on 23 Apr 2021

HAL is a multi-disciplinary open access archive for the deposit and dissemination of scientific research documents, whether they are published or not. The documents may come from teaching and research institutions in France or abroad, or from public or private research centers.
L'archive ouverte pluridisciplinaire HAL, est destinée au dépôt et à la diffusion de documents scientifiques de niveau recherche, publiés ou non, émanant des établissements d'enseignement et de recherche français ou étrangers, des laboratoires publics ou privés. 


\title{
Diabolo Nanoantenna for Enhancing and Confining the Magnetic Optical Field
}

\author{
T. Grosjean, ${ }^{*,+}$ M. Mivelle, ${ }^{\dagger}$ F. I. Baida, ${ }^{\dagger}$ G. W. Burr, ${ }^{\ddagger}$ and U. C. Fischer ${ }^{+, \S}$ \\ ${ }^{\dagger}$ Department of Optics, FEMTO-ST Institute, UMR CNRS 6174, University of Franche-Comté, Besançon, France \\ ${ }^{\ddagger}$ IBM Almaden Research Center, San Jose, California 95120, United States \\ ${ }^{\S}$ Interface Physics Group, Physics Institute, University of Münster, Münster, Germany
}

\begin{abstract}
In this Letter, we introduce a new nanoantenna concept aimed at generating a single magnetic hot spot in the optical frequency range, thus confining and enhancing the magnetic optical field on the background of a much lower electric field. This nanoantenna, designed by applying Babinet's principle to the bowtie nanoaperture, takes the shape of a diabolo. It differs from the well-known bowtie nanoantenna in that the opposing pair of metal triangles are electrically con-
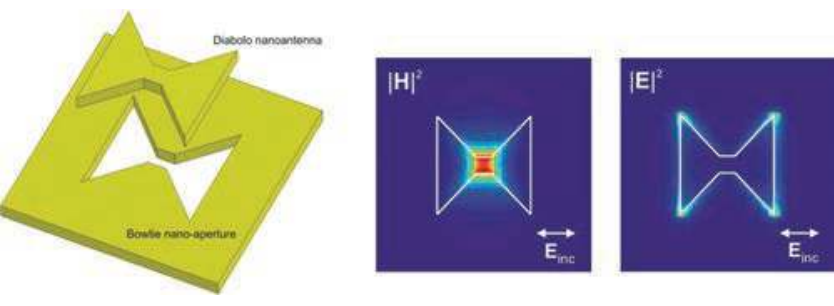
nected through their facing tips. Thus instead of a large charge density accumulating at the air gap of the bowtie nanoantenna, leading to a large electric field, a high optical current density develops within the central "metal gap" of the diabolo nanoantenna, leading to a large magnetic field. Numerical simulation results on the first nanodiabolo geometries show a 2900 -fold enhancement of the magnetic field at a wavelength of $2540 \mathrm{~nm}$, confined to a 40 -by- $40 \mathrm{~nm}$ region near the center of the nanoantenna.
\end{abstract}

KEYWORDS: Optical magnetic field, nanoantenna, plasmon resonance, polarization, electromagnetism

$\mathrm{D}$ epending on their shapes and arrangement, metal nanoparticles have the ability to create dramatically enhanced electromagnetic fields. ${ }^{1}$ An incident wave generates on such structures an electromagnetic near-field which is highly localized in the vicinity of the metal structure. We address here the choice of shapes and arrangement that can create a highly confined and enhanced magnetic field in the optical frequency range on the background of a much lower electric field.

Usually, ferromagnetic materials are used to achieve strongly enhanced magnetic fields. However in the optical frequency regime we have to resort to other means in order to create enhanced magnetic fields. In the electromagnetic near-field, the electric field amplitude may be different from the magnetic field amplitude unlike in a propagating plane wave, where they are equal (in Gaussian units). For instance, in the far field of an electric dipole the electric and magnetic fields have the same amplitude, but in the near-field region, the electric field is much higher for an electric dipole and the magnetic field is much higher for a magnetic dipole. ${ }^{2}$ It is also well-known that for an s-polarized evanescent wave, the magnetic field is larger than the electric field and the opposite is the case for a p-polarized wave. ${ }^{3}$

The ability of some circular nanostructures to develop magnetic moments offers the possibility to locally enhance the magnetic optical near-fields. This was shown for example in the case of apertures of a diameter small compared to the vacuum wavelength in a infinitely conducting film ${ }^{4-6}$ or in a real metal screen, ${ }^{7}$ as well as in the case of metal structures such as rings, ${ }^{8}$ tori, ${ }^{9}$ cylinders, ${ }^{10}$ and split ring resonators. ${ }^{11}$
On the other hand, it is well-known that a large electric field enhancement is created in the air gap between two closely spaced metal nanoparticles, an effect which has long been exploited in the field of surface-enhanced Raman spectroscopy. ${ }^{12}$ Recently the bowtie nanoantenna ${ }^{13,14}$ and bowtie nanoaperture ${ }^{15}$ were shown to be very efficient nanostructures for creating a highly confined and enhanced electric field at optical frequencies in the small gap between their two triangular metal elements. These results lead to a new structure for enhanced magnetic fields through Babinet's principle which relates the scattered electromagnetic field of an aperture in an infinitely thin ideally conducting film and the one of the complementary structure. In its rigorous form, the principle states that the scattered fields are the same if we exchange the electric field of one case with the magnetic field of opposite phase of the other case, for both the exciting and the scattered fields. ${ }^{2}$ It is expected that Babinet's principle holds in an approximate way for real metal films of a finite thickness. In this paper, we examine by numerical calculations the near-field of the complementary bowtie nanoaperture, which we call the "diabolo nanoantenna". Our results show that this structure is capable of significantly enhancing and confining the optical magnetic field.

Principle. The geometry of the proposed 2D diabolo nanoantenna is shown in Figure 1a. Following Babinet's principle 


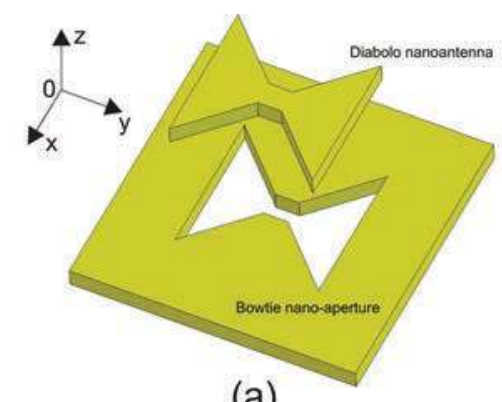

(a)

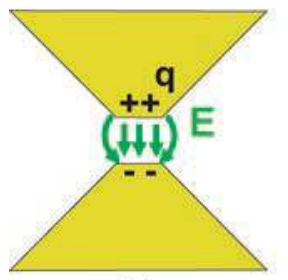

(b)

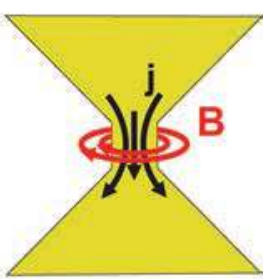

(c)
Figure 1. (a) 3D view of the diabolo nanoantenna and its complementary bowtie nanoaperture. (b, c) Physical principles of the bowtie and diabolo nanoantennas, respectively.

applied to the bowtie nanoaperture, this structure consists of a pair of planar metal triangles joined together through their facing tips. The junction is considered to be a cubic metal particle (called "metal gap" in the following). A magnetic hot spot is generated at the metal gap when the incident electric field is linearly polarized along the axis of symmetry of the nanoantenna that crosses the pair of triangles (called $y$ axis in the following).

The basic principle of the diabolo nanoantenna arises from the electrical connection of two facing metal triangles, which in the bowtie nanoantenna would be electrically isolated. The charge accumulation which occurs at the apexes of the two closely spaced metal triangles of the bowtie nanoantenna (see Figure 1b) is converted into a highly localized enhancement of the optical current density along the metal gap (Figure 1c). The strong capacitive effect which drove the bowtie nanoantenna and led to giant electric-field enhancement is changed to an inductive effect associated with a strong local enhancement of the magnetic field all around the metal gap. The metal triangles act as an electric funnel which reinforces the optical current density into the metal gap. Following Ampère's law, the magnetic field should be azimuthally polarized around this central nanowire. Note that metal conductivity plays a crucial role in the performances of the diabolo nanoantenna since the enhanced magnetic field, which is continuous at the metal interface, can easily penetrate into the metal and lead to noticeable dissipation effects.

Simulation. As a first demonstration of the properties of the diabolo nanoantenna, the electromagnetic response of the structure is simulated using the FDTD method. ${ }^{16}$ The nanoantenna is considered to be deposited onto a dielectric substrate and illuminated with a plane wave propagating inside the substrate at normal incidence (Figure 2).The input electric field is linearly polarized along the $y$ axis.

The model used for the simulations consists of a volume spanning $\pm 2 \mu \mathrm{m}$ in $X$ and $Y$ about the gap of the nanoantenna and -1 to $2 \mu \mathrm{m}$ in $Z$. The refraction index of the substrate is chosen to be equal to 1.5 . A diabolo nanoantenna with $45^{\circ}$ flare angles and a length $D$ of $310 \mathrm{~nm}$ lies on the glass substrate. The electromagnetic response of the structure is studied for various



Figure 2. Scheme of the simulation model under study: (a) top view in the $(X Y)$ plane and $(b)$ side view along the $(X Z)$ plane.

metals (gold, aluminum, and silver), various sizes of the metal gap $(G)$, and thicknesses of the nanoantenna $(T)$. The permittivity of the metals is defined with a Drude model. Note that the height of the metal gap (along the $z$ axis) is given by $T$ whereas $G$ defines both the width and the length of the square nanowire section. All six boundaries of the computation volume are terminated with Convolutional-PMLs ${ }^{17}$ in order to avoid parasitic unphysical reflections around the structure. The nonuniform grid resolution varies from $25 \mathrm{~nm}$ for portions at the periphery of the simulation to $5 \mathrm{~nm}$ in the region of the nanoantenna. The results presented here show the enhancements of the electric and magnetic intensities due to the presence of the diabolo nanoantenna. These enhancements are the field intensities diffracted by the diabolo nanoantenna at a given plane (or position) normalized by the intensities calculated at the same plane (or position) without the presence of the metal structure.

Results and Discussions. Figure 3 shows the near-field distributions of $(a, b)$ the magnetic-field and $(c, d)$ the electricfield enhancements generated by a gold diabolo nanoantenna at a wavelength of $1940 \mathrm{~nm}$. The structure under study is characterized by $G=T=50 \mathrm{~nm}$ and $D=310 \mathrm{~nm}$. Intensity plots are given along $(a, c)$ the longitudinal $(X Z)$ and $(b, d)$ the transverse $(X Y)$ planes. The $(X Z)$ plane crosses the diabolo nanoantenna at the middle of the metal gap whereas the $(X Y)$ plane is taken $10 \mathrm{~nm}$ beneath the nanoantenna output plane, in air.

As predicted, an enhanced magnetic field is highly confined all around the metal gap (Figure $3 \mathrm{a}, \mathrm{b}$ ) and is azimuthally polarized in the (XZ) plane (inset of Figure 3a). The enhancement of the magnetic intensity reaches a value of 230 at a distance of $10 \mathrm{~nm}$ far from the gap (Figure 3b) but becomes higher than 510 very close to the structure (Figure 3a). The enhancement of the electric intensity arises mainly at the end faces of the nanoantenna and is about 35 times smaller than the enhancement of the magnetic intensity at a distance of $10 \mathrm{~nm}$ beyond the metal gap. Figure 3 e shows the normalized intensity of the magnetic field for the $x$ polarization of the incident electric field as compared to the $y$ polarization in Figure $3 \mathrm{~b}$ for otherwise identical conditions. The hot spot at the center of the structure is canceled and the intensity maximum becomes 88 times smaller. These polarization properties allow the magnetic hot spot to be easily and rapidly tuned by rotating the input polarization direction.

Figure 4a reports the magnetic spectral responses of various diabolo nanoantennas of length $D=310 \mathrm{~nm}$ and with parameters $G$ and $T$ equal to 50 and $50 \mathrm{~nm}$ (solid curve), 50 and $20 \mathrm{~nm}$ (dashed curve), 20 and $50 \mathrm{~nm}$ (dotted curve), and 20 and $20 \mathrm{~nm}$ (dashed-dotted curve). Figure $4 \mathrm{~b}$ shows the magnetic response of the structure made of gold (solid curve), aluminum (dashed curve), and silver (dotted curve) for $G=T=50 \mathrm{~nm}$ and $D=$ $310 \mathrm{~nm}$. In all cases, the nanoantennas are excited with a single temporal pulse described by a linearly polarized electric field along the $y$ axis. The time-varying magnetic field is calculated at a point placed $10 \mathrm{~nm}$ far from the center of the metal gap, in air. 



Figure 3. $(a-d)$ Normalized intensity distributions of $(a, b)$ the magnetic field and (c, d) the electric field of a gold diabolo nanoantenna $(G=T=50 \mathrm{~nm}, D=310 \mathrm{~nm})$ excited with a plane wave whose electric field is linearly polarized along the $y$ axis $(\lambda=1940 \mathrm{~nm})$. Intensity plots along $(\mathrm{a}, \mathrm{c})$ the longitudinal $(X Z)$ plane and $(\mathrm{b}, \mathrm{d})$ along the transverse $(X Y)$ plane. Inset of $(a)$ : vectorial map of the magnetic field around the metal gap, in the $(X Z)$ plane. (e) Enhancement of the magnetic intensity for an incident electric field polarized perpendicularly to the $y$ axis. Image size: $700 \mathrm{~nm} \times 700 \mathrm{~nm}$. (f) Same configuration as (a) with $G=T=20$ $\mathrm{nm}$ and $\lambda=2570 \mathrm{~nm}$. Image size: $300 \mathrm{~nm} \times 300 \mathrm{~nm}$.

The spectrum is calculated by a simple Fourier transform of this result and is normalized by the spectrum of the magnetic field at the same position without the metal layer.

These results clearly show that the magnetic hot spot is generated resonantly by the diabolo nanoantenna through the excitation of a plasmon mode. On the one hand, Figure 4a shows that the surface area of the cross section of the metal gap that is perpendicular to the current flow is the key geometrical parameter in the definition of the amplitude of the enhanced magnetic field. Any decrease in the surface area leads to an increase of the magnetic field and two geometries of the metal gap that lead to the same surface generate magnetic effects of the same level but not at the same wavelength (see dashed and dotted curves of Figure 4a). Thus, the maximum enhancement of the magnetic intensity, which is about 510 for a gold diabolo nanoantenna with $G=T=50 \mathrm{~nm}$ Figure (3a), becomes higher than 2900 when $G=$ $T=20 \mathrm{~nm}$ (see Figure 3f). Note that the enhancement of the magnetic field in circular apertures small compared to the wavelength is instead a decreasing function of the aperture size. ${ }^{4}$ On the other hand, an increase of metal conductivity induces stronger current density in the structure and therefore higher inductive effect in the gap zone. Figure $4 \mathrm{~b}$ shows that the enhancement of the magnetic intensity is better for silver than for gold and aluminum, which have lower conductivities in
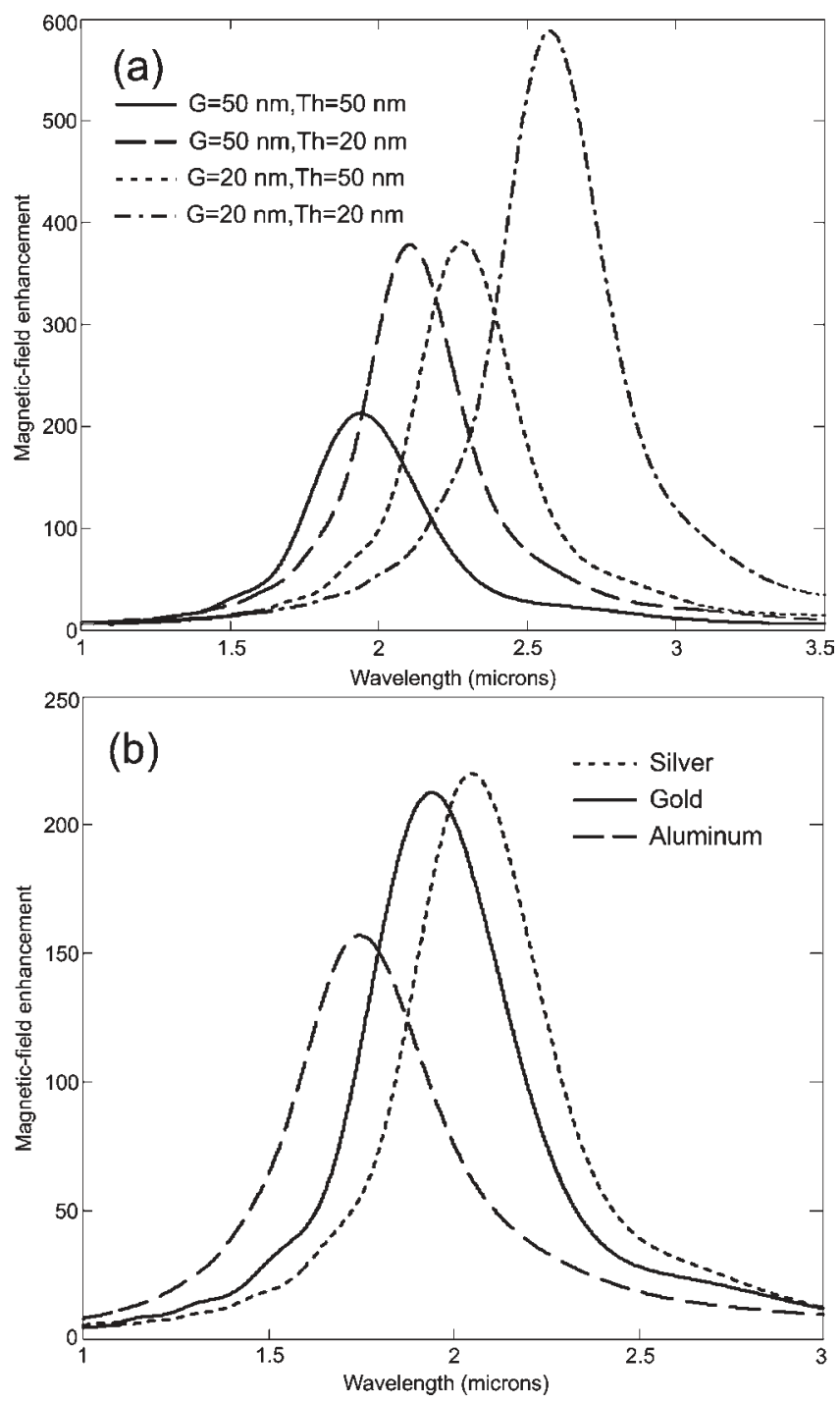

Figure 4. (a) Comparison of the magnetic spectral responses of gold diabolo nanoantennas with $D=310 \mathrm{~nm}$ and $G$ and $T$ equal to 50 and $50 \mathrm{~nm}$ (solid curve), 50 and $20 \mathrm{~nm}$ (dashed curve), 20 and $50 \mathrm{~nm}$ (dotted curve), and 20 and $20 \mathrm{~nm}$ (dashed-dotted curve). (b) Magnetic spectral responses of diabolo nanoantennas made of gold (solid line), aluminum (dashed line), and silver (dotted line) with $G=50 \mathrm{~nm}, T=$ $50 \mathrm{~nm}$, and $D=310 \mathrm{~nm}$.

the near-infrared region. When the cross section of the metal gap becomes smaller than twice the skin depth, resistivity increases and produces a higher electric field in and around (by continuity) the optical nanowire. We see in panels a and $f$ of Figure 3 that the enhanced magnetic field does not show a constant amplitude all around the metal gap. The different dielectric constants at both sides of the diabolo nanoantenna (the structure lies on a glass substrate) and the asymmetric illumination can contribute to this phenomenon. However, there is no simple interpretation of the fact that the higher magnetic field is located at the glass/metal interface in Figure 3a and at the metal/air interface in Figure 3f, and this phenomenon will have to be investigated in more detail.

The underlying physics of the diabolo nanoantenna is that of a short-circuit between two oppositely charged metal bodies. Under illumination with an electric field polarized along the antenna $y$ axis, charge accumulations of opposite signs arise at the 


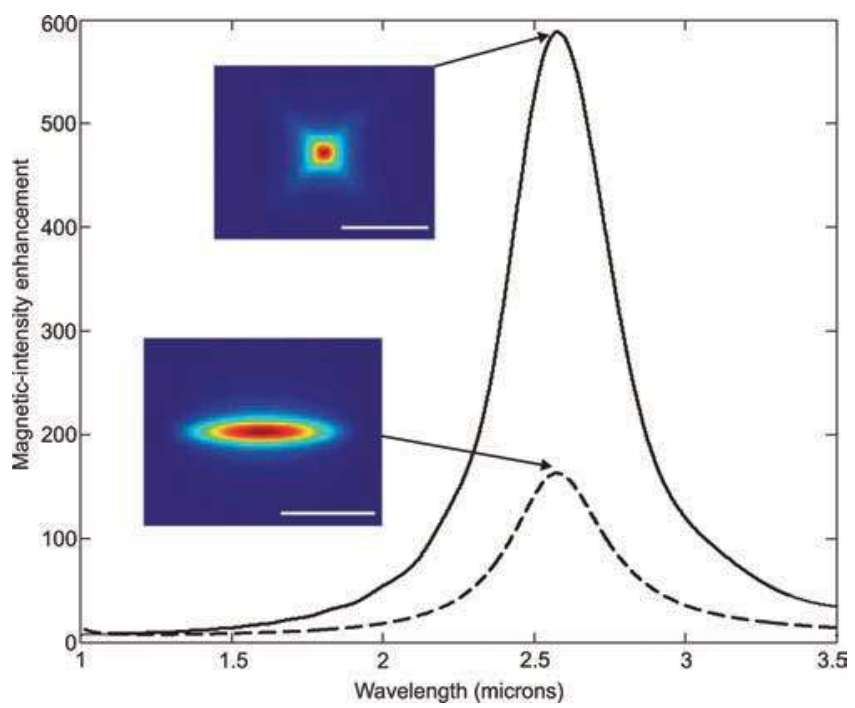

Figure 5. Comparison of the magnetic spectral responses of a gold diabolo nanoantenna with $D=310 \mathrm{~nm}$ and $G=T=20 \mathrm{~nm}$ (solid curve) and a $455 \mathrm{~nm}$ long gold nanowire of $20 \mathrm{~nm}$ large square cross section (dashed curve). Figure insets: transverse distributions of the magnetic intensity simulated at the common resonance wavelength of the two structures (scale bar $200 \mathrm{~nm}$ ).

end faces of the structure. These charge effects lead to high enhancements of the electric field on both sides of the diabolo nanoantenna, as shown in Figure 3d. The resulting potential difference between these two electrically connected metal structures generates currents which are guided across the metal gap. The double-funnel geometry of the diabolo nanoantenna concentrates and amplifies with high efficiency the current density in the metal gap. Following Ampère's law, the resulting local increase of the current intensity leads to a strong enhancement of the surrounding magnetic field (as shown in Figure 3a,b). Note that the charge accumulations on the side edges of the structure, the currents into the metal gap, and the metal conductivity may find a direct relationship with the capacitance, inductance and resistance of an equivalent RLC circuit, respectively. Figure 5 shows a comparative study of the magnetic spectral responses of a gold diabolo nanoantenna with $G=T=20 \mathrm{~nm}$ and $D=310 \mathrm{~nm}$ and a $455 \mathrm{~nm}$ long gold nanowire whose square cross section is $20 \mathrm{~nm}$ large. Figure insets represent the transverse distributions of the magnetic intensity, simulated $10 \mathrm{~nm}$ beyond the structures, at their common resonance wavelength of $2570 \mathrm{~nm}$ (scale bar $=200$ $\mathrm{nm}$ ). These results show an enhancement 4-fold smaller for the nanowire than for the diabolo nanoantenna with a loss of confinement along the nanowire main axis, indicating the importance of the triangular portions of the diabolo antenna.

The highly confined distribution of the magnetic field around the metal gap (Figure 3a) shows that the plasmon mode sustained by the diabolo nanoantenna is associated with an effective wavelength much smaller than the vacuum wavelength. This may explain why the plasmon mode is excited at a length of the diabolo nanoantenna considerably smaller than the vacuum wavelength. This property could be due to the triangular geometries of the diabolo nanoantenna since triangular and conical structures are known to sustain plasmon modes of very small wavelengths, leading for example to the superfocusing effect. ${ }^{18}$

While the 2900-fold enhancement of the optical magnetic field offered by the diabolo nanoantenna is large, it is significantly smaller than the typical electric field enhancements offered by the bowtie nanoantenna. ${ }^{19}$ In order to explain the more modest resonance enhancement offered by the diabolo nanoantenna, we have to consider the different boundary conditions. For the diabolo nanoantenna, the largest magnetic fields are tangential to the metal surface, while at the tips of the bowtie structure, the largest electric fields are perpendicular to the metal surface. Since the perpendicular electric field has a discontinuity across the interface, the electric field in the metal is smaller than the field just outside and thus the losses in the metal are small. On the other hand, the tangential magnetic field is continuous across the interface and high magnetic fields exist in the metal leading to stronger losses. For this reason only a moderate resonance enhancement can be obtained for diabolo nanoantenna as compared to the bowtie nanoantenna. The losses in the metal are associated with imaginary part of the dielectric constant, which tends to increase with decreasing wavelength. Therefore the resonance enhancement gets smaller and smaller with decreasing wavelength.

Conclusion. In conclusion we have shown that the diabolo nanoantenna is an interesting structure for creating a highly localized magnetic field with a high field enhancement in the near-infrared region. Structures of this type can be readily fabricated, either by a focused ion beam or electron beam lithography, similar to the way the dipole and bowtie nanoantennas were produced. ${ }^{20,21}$ Alternately, modified nanosphere lithography could be employed. ${ }^{22}$ The existence of an enhanced magnetic field can be tested by using a SNOM configuration which allows us to measure the vectorial magnetic and electric nearfields. ${ }^{23,24}$ We intend to use such structures as a detector for the optical magnetic field and for the magneto-optic modulation of plasmon polaritons at near-infrared or even visible frequencies, which is not possible by using ferromagnets. ${ }^{25}$ The diabolo nanoantenna may be also relevant for inducing magnetic transitions in matter. ${ }^{26}$

\section{AUTHOR INFORMATION}

\section{Corresponding Author}

*E-mail: thierry.grosjean@univ-fcomte.fr.

\section{ACKNOWLEDGMENT}

This work is funded by the "Agence Nationale de la Recherche" under Contract No. ANR07-NANO-036. It is supported by the "Pôle de compétitivité Microtechnique".

\section{REFERENCES}

(1) Novotny, L.; Hecht, B. Principles of nano-optics; Cambridge University Press: Cambridge, U.K., and New York, 2006.

(2) Jackson, J. Classical Electrodynamics; John Wiley \& Sons, Inc.: New York, 1999.

(3) Fischer, U. C. In Scanning tunneling Microscopy and Related Methods; Behm, R. J., Garcia, N., Rohrer, H., Eds.; Proceedings of the NATO ASI Series E; Kluwer Academic: Dordrecht, Boston, and London, 1990; Vol. 184; pp 475-496.

(4) Bethe, H. Phys. Rev. 1944, 66, 163-182.

(5) Bouwkamp, C. Rep. Phys. 1954, 27, 35-100.

(6) Samii, Y. R.; Mittra, R. IEEE Trans. Antennas Propag. 1977, 25, 180-187.

(7) Bortchagovsky, E.; des Francs, G. C.; Molenda, D.; Naber, A.; Fischer, U. Appl. Phys. B: Laser Opt. 2006, 84, 49-53. 
(8) Suarez, M.; Grosjean, T.; Charraut, D.; Courjon, D. Opt. Commun. 2007, 270, 447-454.

(9) Mary, A.; Dereux, A.; Ferrell, T. Phys. Rev. B 2005, 72, No. 155426.

(10) Schröter, U.; Dereux., A. Phys. Rev. B 2001, 64, No. 125420.

(11) Zhou, J.; Koschny, T.; Kafesaki, M.; Economou, E. N.; Pendry, J. B.; Soukoulis, C. M. Phys. Rev. Lett. 2005, 95, No. 223902.

(12) Moskovits, M. Rev. Mod. Phys. 1985, 57, 783-826.

(13) Grober, R.; Schoelkopf, R; Prober, D. Appl. Phys. Lett. 1997, 70, 1354-1356.

(14) Schuck, P.; Fromm, D.; Sundaramurthy, A.; Kino, G.; Moerner, W. Phys. Rev. Lett. 2005, 94, No. 017402.

(15) Wang, L.; Uppuluri, S.; Jin, E.; Xu, X. Nano Lett. 2006, 6, 361.

(16) Taflove, A.; Hagness, S. Computational Electrodynamics: The Finite-Difference Time-Domain Method, 3rd ed.; Artech House: Boston, 2005.

(17) Roden, J.; Gedney, S. Microwave Opt. Technol. Lett. 2000, 27, 334-339.

(18) A.J. Babadjanyan, K. N.; N.L. Margaryan J. Appl. Phys. 2000, 87, 3785-3788.

(19) Fischer, H.; Martin, O. Opt. Express 2008, 16, 9144-9154.

(20) Mühlschlegel, P.; Eisler, H.-J.; Martin, O.; Hecht, B.; Pohl, D. Science 2005, 308, 1607-1609.

(21) Farahani, J.; Eisler, H.-J.; Pohl, D.; Pavius, M.; Flackiger, P.; Gasser, P.; Hecht, B. Nanotechnology 2007, 18, No. 125506.

(22) Fischer, U.; Heimel, J.; Maas, H.-J.; Hartig, M.; Hoeppener, S.; Fuchs, H. Surf. Interface Anal. 2002, 33, 75-80.

(23) Burresi, M.; van Oosten, D.; Kampfrath, T.; Schoenmaker, H.; Heideman, R.; Leinse, A.; Kuipers, L. Science 2009, 326, 550-553.

(24) Grosjean, T.; Ibrahim, I. A.; Suarez, M. A.; Burr, G. W.; Mivelle, M.; Charraut, D. Opt. Express 2010, 18, 5809-5824.

(25) Temnov, V. V.; Armelles, G.; Woggon, U.; Guzatov, D; Cebollada, A; Garcia-Martin, A.; Garcia-Martin, J.-M.; Thomay, T.; Leitenstorfer, A.; Bratschitsch, R. Nat. Photonics 2010, 4, 104-111.

(26) Karaveli, S.; Zia, R. Opt. Lett. 2010, 35, 3318-3320. 